\title{
High serum levels of 8-OHdG are an independent predictor of post-stroke depression in Chinese stroke survivors
}

This article was published in the following Dove Press journal: Neuropsychiatric Disease and Treatment

\section{Zhihua Liu \\ Yan Cai \\ Jincai $\mathrm{He}$}

Department of Neurology, The First Affiliated Hospital of Wenzhou Medical University, Wenzhou, China
Correspondence: Jincai He

Department of Neurology, The First

Affiliated Hospital of Wenzhou Medical University, No 2 Shifu Lane, Wenzhou,

Zhejiang Province 325000, China

Tel/fax +8657755579363

Email hjc@wmu.edu.cn
Purpose: Although previous studies have investigated oxidative stress biomarkers in association with depression in non-stroke subjects, the association between oxidative deoxyribonucleic acid damage and post-stroke depression (PSD) remains unelucidated.

Patients and methods: Two hundred forty-one first-ever ischemic stroke patients were consecutively recruited within the first $24 \mathrm{~h}$ of stroke onset and were followed up at 1 month. Serum 8-hydroxy-2'-deoxyguanosine $(8-\mathrm{OHdG})$ and catalase (CAT) levels were measured within $24 \mathrm{~h}$ of admission using a commercially available enzyme-linked immunosorbent assay. The 17-item Hamilton Depression Scale was used to evaluate depressive symptoms. Diagnosis of PSD was made in line with the Diagnostic and Statistical Manual of Mental Disorders, 4th edition criteria for depression.

Results: Serum levels of $8-\mathrm{OHdG}(P<0.001)$ and CAT $(P=0.025)$ increased in depressed patients at admission. A positive correlation was found between the 8-OHdG and CAT levels in both the total stroke patients $(r=0.320, P<0.001)$ and the depressed patients $(r=0.300$, $P=0.012$ ). The 8 -OHdG levels were positively correlated with the 17-item Hamilton Depression Scale scores $(r=0.129, P=0.046)$ in depressed patients. Multivariate analyses found that 8-OHdG levels $\geq 200.0 \mathrm{ng} / \mathrm{L}$ were independently associated with PSD (odds ratio, 7.477; 95\% CI, $3.342-16.289, P<0.001$ ) after adjusting for possible relevant confounders.

Conclusion: Higher serum 8-OHdG levels at admission were found to be correlated with PSD 1 month after stroke.

Keywords: oxidative stress, 8-hydroxy-2'-deoxyguanosine, catalase, depression, stroke

\section{Introduction}

Post-stroke depression (PSD) is a highly prevalent neuropsychiatric sequela of stroke that acts as an important index of both functional and treatment outcomes in patients. PSD can interfere with stroke recovery and is associated with the severity of stroke, cognitive impairment, and increased mortality. ${ }^{1-3}$ Therefore, early diagnosis and intervention in PSD are beneficial to the functional recovery of stroke patients. However, research into the etiological mechanisms of PSD in clinical populations is limited.

Accumulating evidence has suggested that ischemic stroke is intimately linked to acute bursts of reactive oxygen species (ROS) or reactive nitrogen species (RON), and oxidative deoxyribonucleic acid (DNA) damage appears to be involved in the pathogenesis of brain ischemia. ${ }^{4}$ Antioxidants can remove ROS and RON through scavenging radicals and suppressing the oxidative stress pathway, further preventing oxidative damage to neurons. ${ }^{5}$ An imbalance between the generation of ROS by pro-oxidants and the defense mechanisms against ROS by antioxidants can elicit 
pathological changes such as lipid peroxidation, DNA damage, autoimmune responses, and protein carbonylation..$^{6,7}$ Catalase (CAT) serves as an intracellular antioxidant enzyme that metabolizes ROS into less toxic molecules and catalyzes hydrogen peroxide into water and oxygen. ${ }^{8}$ Previous studies have reported that serum or plasma CAT levels were significantly lower in acute ischemic stroke (AIS) patients than in controls. ${ }^{9,10}$ A recent longitudinal observational study of stroke patients demonstrated decreased serum CAT levels in stroke patients compared with controls within $24 \mathrm{~h}$ of the onset of ischemia, and its levels did not change during the subsequent 21 days. $^{9}$

Oxidative DNA damage has been proposed to be a causative factor in blood-brain barrier dysfunction and is known to induce neuronal degeneration and apoptosis. ${ }^{11,12}$ Damage to DNA is indicated by increased levels of 8-hydroxy-2'deoxyguanosine (8-OHdG), a repair product of the oxidation of guanine in DNA. ${ }^{13} 8-\mathrm{OHdG}$ can be detected in human tissue, blood samples, or urine and is considered a reliable and pivotal biomarker of generalized and cellular oxidative stress. ${ }^{14,15}$ Previous studies have reported that peripheral 8-OHdG is a valuable indicator of the severity of oxidative brain damage in acute cerebral infarction. ${ }^{16}$ The role of 8-OHdG as a potential and predictive biomarker of atherosclerosis, cardiovascular disease, neurodegenerative disorders, such as Alzheimer's disease and Parkinson's disease, and psychiatric disorders, such as schizophrenia, has been well recognized. ${ }^{17-19}$ Elevated levels of 8-OHdG have been reported to be correlated with clinical outcomes of stroke and have a close relationship with atherosclerotic plaque types and vascular recurrence in non-cardioembolic stroke patients. ${ }^{13,20,21}$ Recently, Myoren et al found that increased 8-OHdG might be able to powerfully predict cardiovascularrelated death. ${ }^{22}$

Previous studies have assessed the role of CAT or 8-OHdG in patients with depression, but these studies were controversial. While some authors have reported increased CAT activity in depressed patients, several studies have revealed no significant changes compared with healthy controls. $^{8,23}$ Additionally, lower gene expression of CAT was noted in patients with major depression. ${ }^{24}$ Although the majority of studies have identified that levels of $8-\mathrm{OHdG}$ increased in depressed patients, a recent study of a large adult cohort found no increased levels of $8-\mathrm{OHdG} .^{25-27}$

Thus, it is reasonable that oxidative stress biomarkers and levels of antioxidant enzymes may have a predictive role in PSD. The findings of a recent study suggested that the activity of CAT in erythrocytes is negatively correlated with Geriatric Depression Scale scores during a brain stroke. ${ }^{6}$ However, no study to date has investigated the relationship between levels of oxidative DNA damage and PSD. Furthermore, the results of a study of a multiracial-ethnic stroke cohort suggested that racial-ethnic differences might have a role in the prevalence of depression among stroke survivors. ${ }^{28}$ The main aim of our study was to explore if high levels of 8-OHdG contribute to the development of PSD in Chinese subjects who suffered an AIS.

\section{Patients and methods \\ Patients and study design}

Two hundred forty-one patients who were consecutively admitted to the Stroke Unit of the First Affiliated Hospital of Wenzhou Medical University within the first $24 \mathrm{~h}$ of first-ever ischemic stroke onset between October 2013 and September 2014 were enrolled in this study.

Participants were excluded if the following criteria were identified: a history of depression (clinical diagnosis or previous treatment) or other psychiatric disorders; a history of psychiatric treatment; use of antioxidants drugs or vitamin supplementation within the prior 30 days; an application of thrombolytic therapy; implant of a carotid or coronary stent or other major surgical interventions; a medical history of pre-stroke dementia; significant acute or chronic inflammatory factors or neurological illnesses other than stroke; severe aphasia or dysarthria; major medical illness; and age $<18$ years. A flow chart of the study design is shown in Figure S1.

The study was approved by the Medical Ethics Committee of the First Affiliated Hospital of Wenzhou Medical University with the following reference number: 201340. All participants provided written informed consent prior to participation in accordance with the Declaration of Helsinki of 1975.

\section{Neuropsychiatric assessment}

The severity of depressive symptoms at admission (baseline) was measured with the 17-item Hamilton Depression Scale (HAMD-17) within $24 \mathrm{~h}$ of admission. ${ }^{29}$ The HAMD-17 has been the gold standard in depression trials since its introduction in 1960 by Hamilton. ${ }^{30}$ Numerous studies have examined the high validity and utility of the HAMD-17 as a useful screening tool for depressive symptoms, and the optimal cutoff point has been found to be 7 in the general population or in patients with neurological diseases such as stroke, epilepsy, and severe traumatic brain injury. ${ }^{31-34}$

Patients with possible depressive symptoms at 1 month after onset of the neurological symptoms of the acute stroke were further interviewed with the Structured Clinical 
Interview of the Diagnostic and Statistical Manual of Mental Disorders, 4th edition (DSM-IV), for the diagnosis of PSD. ${ }^{35}$ One of the trained psychologist researchers administered the DSM-IV, and 2 neuropsychologists conducted the psychiatric rating scales.

\section{Functional and neurological assessment}

Acute stroke was confirmed by cranial CT or diffusionweighted MRI in all enrolled patients. Upon admission to the Stroke Unit, an expert neurologist performed a full neurological evaluation of the patients and recorded the type and location of the index stroke. The quantitative evaluation of stroke-induced neurological deficits during the acute phase was conducted using the National Institutes of Health Stroke Scale (NIHSS) at admission. An NIHSS score $\leq 3$ is defined as minor stroke, and $>3$ as moderate-to-severe stroke. ${ }^{36,37}$ The Barthel index (BI) and the Mini-Mental State Exam (MMSE) were used to assess functional and cognitive impairment, respectively, at discharge. ${ }^{38-40}$

\section{Serum 8-OHdG and CAT measurement}

Blood was collected from the antecubital vein within $24 \mathrm{~h}$ of admission. Blood samples were centrifuged at 3,000 rpm for $10 \mathrm{~min}$, and the plasma was separated and aliquots of the samples were immediately stored at $-80^{\circ} \mathrm{C}$ before the assays. The samples were thawed only once before the analyses. The serum 8-OHdG and CAT antibody concentrations were measured by a commercially available enzyme-linked immunosorbent assay (Xinfan Biotechnology Co., Ltd, Shanghai, China). All procedures used in the present study followed the instructions strictly. The optical density value was measured with a microplate reader at a wavelength of $450 \mathrm{~nm}$. Then, we calculated the concentration of 8-OHdG and CAT according to the standard curve. All standards and samples were tested in duplicate wells. The inter-assay and intra-assay coefficients of variation for $8-\mathrm{OHdG}$ were $11 \%$ and $9 \%$, respectively. The mean minimum detectable dose of human $8-\mathrm{OHdG}$ was $10 \mathrm{ng} / \mathrm{L}$, and the line range was 10-300 ng/L. The inter-assay and intra-assay coefficients of variation for human CAT were $11 \%$ and $9 \%$, respectively, and the mean minimum detectable dose was $3 \mathrm{U} / \mathrm{L}$. The measurement range of the human CAT kit was 3-90 U/L.

\section{Statistical analysis}

Categorical data were recorded as relative and absolute frequencies. Continuous variables were tested for distribution using the Kolmogorov-Smirnov test. The normally distributed continuous variables were analyzed with Student's $t$-test or analysis of variance and are described as the mean $\pm \mathrm{SD}$, whereas the asymmetrically distributed variables were analyzed with the Mann-Whitney $U$ test or the KruskalWallis test and are expressed as the median interquartile range. Correlations between $8-\mathrm{OHdG}$ and the variables were assessed using Spearman's correlation coefficient.

A receiver-operating characteristic (ROC) curve analysis was used to determine the diagnostic accuracy and optimal cutoff values of $8-\mathrm{OHdG}$ in the diagnosis of PSD. The area under the curve (AUC) was provided with a 95\% CI, which was obtained by using MedCalc 12.5 (MedCalc Software, Ostend, Belgium). We treated the serum 8-OHdG level as a dichotomized variable (by the optimal cutoff) in the statistical analyses. The influence of the serum 8-OHdG levels on PSD was estimated by binary logistic regression analysis, after adjusting for potential confounders regarded as being clinically relevant. A 2 -sided $P$-value $<0.05$ was regarded as statistically significant. All data analyses were performed using SPSS version 22.0 software (SPSS Inc., Chicago, IL, USA).

\section{Results}

The study cohort consisted of 552 patients at baseline. By the time of the follow-up at 1 month, 241 patients remained in our study. However, these 241 patients were similar in terms of their baseline characteristics such as age, sex, and NIHSS scores (all $P>0.05$ ) compared with the overall cohort. The baseline clinical characteristics of enrolled patients with AIS are shown in Tables 1, 2, and S1.

The occurrence of PSD was 29.0\%. Patients with PSD had more severe strokes $(P<0.001)$, and poorer functional outcomes $(P=0.013)$ and cognitive functioning $(P=0.018)$ than those without PSD.

The serum 8-OHdG levels of all stroke patients and patients with PSD and without PSD at admission were 183.0 $\pm 59.8,218.0$ (170.6-246.7), and 164.8 (121.1-208.0) ng/L (Figure 1), respectively. The serum 8-OHdG levels at baseline were significantly higher in patients with PSD than in patients without PSD at 1 month $(P<0.001$, Tables 1 and S2). A positive correlation was found between the $8-\mathrm{OHdG}$ and CAT levels in both the total stroke patients ( $r=0.320, P<0.001$, Figure 2$)$ and the depressed patients ( $r=0.300, P=0.012$ ). 8-OHdG was positively correlated with the HAMD scores $(r=0.129, P=0.046)$ in depressed patients. No significant differences in serum CAT or 8 -OHdG levels were identified between the minor stroke and the moderate-to-severe stroke groups (Table S3). Statistical analysis revealed no influence of age, NIHSS scores (Table S4), or BI scores on 8-OHdG levels in stroke patients (all $P>0.05)$. 
Table I Baseline characteristics of the studied sample

\begin{tabular}{|c|c|c|c|}
\hline Baseline characteristics & PSD $(n=70)$ & No-PSD $(n=|7|)$ & $P$-value \\
\hline \multicolumn{4}{|l|}{ Demographic characteristics } \\
\hline Female, \% & $25(35.7)$ & $61(35.7)$ & 0.995 \\
\hline Age (years), median (IQR) & $64(57-7 I)$ & $64(56-70)$ & 0.732 \\
\hline Years of education (years), median (IQR) & $3(0-6)$ & $3(0-6)$ & 0.990 \\
\hline $\mathrm{SBP}(\mathrm{mmHg})$, mean $\pm \mathrm{SD}$ & $156.7 \pm 20.2$ & $153.3 \pm 22.6$ & 0.285 \\
\hline $\mathrm{DBP}(\mathrm{mmHg})$, mean $\pm \mathrm{SD}$ & $84.4 \pm 12.1$ & $81.0 \pm 13.1$ & 0.061 \\
\hline $\mathrm{BMI}, \mathrm{kg} / \mathrm{m}^{2}$, mean $\pm \mathrm{SD}$ & $24.5 \pm 3.7$ & $24.2 \pm 3.0$ & 0.489 \\
\hline \multicolumn{4}{|c|}{ Prescribed drugs used chronically prior to the ischemic events } \\
\hline Hypertension medicine use, n (\%) & $36(51.4)$ & $95(55.6)$ & 0.559 \\
\hline Diabetes medicine use, $\mathrm{n}(\%)$ & $10(14.3)$ & $33(19.3)$ & 0.356 \\
\hline Lipid-lowering medicine use, $\mathrm{n}(\%)$ & 0 & $4(2.3)$ & 0.462 \\
\hline Aspirin or clopidogrel use, n (\%) & $4(5.7)$ & $9(5.3)$ & 1.000 \\
\hline \multicolumn{4}{|l|}{ Stroke etiology, n (\%) } \\
\hline Atherosclerosis & $6 I(87.1)$ & $156(9 \mid .2)$ & 0.098 \\
\hline Cardioembolism & $4(5.7)$ & $8(4.7)$ & \\
\hline Small vessel occlusion & $3(4.3)$ & $7(4.1)$ & \\
\hline Unknown & $2(2.9)$ & 0 & \\
\hline \multicolumn{4}{|l|}{ Lesion location (\%) } \\
\hline Frontal lobe & $6(8.6)$ & $28(16.4)$ & 0.106 \\
\hline Parietal lobe & $\mathrm{I}(\mathrm{I} .4)$ & $7(4.1)$ & \\
\hline Temporal lobe & $\mathrm{I}(\mathrm{I} .4)$ & $7(4.1)$ & \\
\hline Occipital lobe & $3(4.3)$ & $7(4.1)$ & \\
\hline Basal ganglia & $24(34.3)$ & $43(25.1)$ & \\
\hline Brainstem & $4(5.7)$ & $21(12.3)$ & \\
\hline Cerebellum & $5(7.1)$ & $6(3.5)$ & \\
\hline Thalamus & $4(5.7)$ & $19(11.1)$ & \\
\hline Other & $22(31.5)$ & $33(19.3)$ & \\
\hline \multicolumn{4}{|l|}{ Vascular risk factors, $\mathbf{n}(\%)$} \\
\hline Hypertension & $48(68.6)$ & III (64.9) & 0.586 \\
\hline Diabetes mellitus & $16(22.9)$ & $37(21.6)$ & 0.836 \\
\hline Coronary artery disease & 0 & $8(4.7)$ & 0.149 \\
\hline Hyperlipidemia & $\mathrm{I}(\mathrm{I} .4)$ & II (6.4) & 0.195 \\
\hline Smokers & $21(30.0)$ & $50(29.2)$ & 0.906 \\
\hline Alcohol consumers & $23(32.9)$ & $64(37.4)$ & 0.503 \\
\hline Stroke severity, median NIHSS score (IQR) & $4(2-6)$ & $2(1-3)$ & $<0.001 * * *$ \\
\hline NIHSS $\leq 3$ & $34(48.5)$ & $130(76.0)$ & $<0.001 * * *$ \\
\hline Cognitive function, median MMSE score (IQR) & $19(15-25)$ & $23(18-26)$ & $0.018 *$ \\
\hline Bl score, median (IQR) & $72.5(50-100)$ & $95(60-100)$ & $0.013 *$ \\
\hline \multicolumn{4}{|l|}{ Laboratory parameters } \\
\hline 8-OHdG (ng/L), median (IQR) & $218.0(170.6-246.7)$ & I64.8 (I2I.I-208.0) & $<0.00 \mathrm{I}^{* * *}$ \\
\hline $\mathrm{CAT}(\mathrm{U} / \mathrm{L})$, mean $\pm \mathrm{SD}$ & $64.1 \pm 23.9$ & $57.3 \pm 20.1$ & $0.025^{*}$ \\
\hline FBG (mmol/L), median (IQR) & $5.3(4.3-7.8)$ & $4.8(4.3-5.9)$ & $0.04 *$ \\
\hline TG (mmol/L), median (IQR) & $4.9(4.2-5.7)$ & $4.6(3.9-5.4)$ & 0.158 \\
\hline TC (mmol/L), median (IQR) & $1.8(1.3-2.3)$ & $1.5(1.2-2.0)$ & 0.231 \\
\hline HDL-C (mmol/L), median (IQR) & $1.1(0.9-1.3)$ & $1.1(0.9-1.3)$ & 0.492 \\
\hline LDL-C (mmol/L), median (IQR) & $2.8(2.2-3.7)$ & $2.7(2.1-3.4)$ & 0.400 \\
\hline
\end{tabular}

Notes: Continuous variables are expressed as the mean \pm SD or the median (IQR). Categorical values are given as frequencies (percentages). The $P$-values reflect comparisons between PSD group and non-PSD group. $*<0.05$, $* * *<0.001$, vs PSD group by univariate.

Abbreviations: 8-OHdG, 8-hydroxy-2'-deoxyguanosine; BI, modified Barthel index; BMI, body mass index; CAT, catalase; DBP, diastolic blood pressure; FBG, fasting blood glucose; HDL-C, high-density lipoprotein cholesterol; IQR, interquartile range; LDL-C, low-density lipoprotein cholesterol; MMSE, mini-mental state examination; NIHSS, National Institutes of Health Stroke Scale; PSD, post-stroke depression; SBP, systolic blood pressure; TC, total cholesterol; TG, triglyceride.

In the logistic regression analysis, $8-\mathrm{OHdG}$, taken as a continuous variable, was independently associated with PSD (OR, 1.019; 95\% CI, 1.012-1.027, $P<0.001$, Table 2) after being adjusted for age, sex, NHISS scores, MMSE scores,
BI scores, current smoking status, current drinking status, and fasting blood glucose and CAT levels.

Based on the ROC curve, the optimal cutoff 8 of serum 8-OHdG levels as an indicator for the prediction of PSD 
Table 2 Adjusted OR of depression for 8-OHdG levels in stroke patients

\begin{tabular}{lll}
\hline Variables & OR $(95 \% \mathrm{CI})$ & $P$-value \\
\hline Age & $1.006(0.965-1.048)$ & 0.779 \\
Sex & $0.728(0.292-1.818)$ & 0.497 \\
NIHSS score & $1.288(1.099-1.51 \mathrm{I})$ & $0.002^{* *}$ \\
MMSE score & $0.931(0.870-0.996)$ & $0.038^{*}$ \\
BI score & $1.002(0.985-1.020)$ & 0.795 \\
Smokers & $1.379(0.568-3.350)$ & 0.477 \\
Alcohol consumers & $0.745(0.332-1.673)$ & 0.476 \\
CAT & $1.007(0.991-1.024)$ & 0.383 \\
FBG & $1.198(1.020-1.407)$ & $0.027^{*}$ \\
Hypertension & $1.385(0.642-2.991)$ & 0.406 \\
Diabetes mellitus & $0.566(0.205-1.559)$ & 0.271 \\
Statins at admission & $1.979(0.139-28.158)$ & 0.614 \\
Vitamin C at admission & $0.722(0.160-3.255)$ & 0.672 \\
8-OHdG level at admission & $1.019(1.012-1.027)$ & $<0.00$ I*** \\
8-OHdG level at admission & $7.477(3.432-16.289)$ & $<0.00$ I*** \\
>200.0 ng/L & & \\
\hline Note & &
\end{tabular}

Note: $* P<0.05, * * P<0.01$, $* * * P<0.001$.

Abbreviations: 8-OHdG, 8-hydroxy-2'-deoxyguanosine; Bl, Barthel index; CAT, catalase; FBG, fasting blood glucose; MMSE, mini-mental state examination; NIHSS, National Institutes of Health Stroke Scale; OR, odds ratio.

was projected to be $200.0 \mathrm{ng} / \mathrm{L}$, which yielded a sensitivity of $65.7 \%$ and a specificity of $71.0 \%$, with an AUC of 0.723 (95\% CI, 0.656-0789, $P<0.001$, Figure 3). Furthermore, there was an increased risk of PSD associated with serum 8-OHdG levels $\geq 200.0 \mathrm{ng} / \mathrm{L}$ (odds ratio [OR], 7.477; 95\% CI, 3.432-16.289, $P<0.001)$.

\section{Discussion}

To the best of our knowledge, this is the first study that explored the relationship between serum 8-OHdG levels and depression in stroke patients at 1 month post-stroke.

In the present study, we found that the prevalence of PSD was $29.0 \% 1$ month after stroke, which was in agreement with the results of recent studies. A prior meta-analysis of
50 studies, which included 20,293 patients, reported that the pooled prevalence of PSD was $29 \%$ at any time point within 5 years. ${ }^{41}$ We also found that the disability severity was related to PSD. Impairment in activities of daily living has been consistently identified as a risk factor for PSD. ${ }^{3}$ Similarly, Matsuzaki et al found that depression occurred independently after stroke and could individually influence functional recovery. ${ }^{42}$

Our main findings indicated that elevated serum 8-OHdG is an independent predictor for the development of PSD at 1 month. Similarly, 1 previous study indicated strong evidence that serum $8-\mathrm{OHdG}$ increased significantly in participants with major depression, and participants with recurrent episodes of depression had more DNA damage than those with single episodes. ${ }^{43}$ Increased levels of oxidative stress biomarkers have been demonstrated in subjects with a history of suicide attempts. ${ }^{44}$ A recent clinical study demonstrated that the association between the presence of depressive symptoms and 8-OHdG levels is independent of the menstrual cycle. ${ }^{45}$ While some studies have reported increased 8-OHdG levels in depression, 1 study reported lower levels. ${ }^{25,26}$ This discrepancy may have resulted from differences in the biological specimens, laboratory techniques, or the time of assessment used in the studies. ${ }^{27}$

The role of serum 8-OHdG in the pathophysiology of PSD remains unknown. Many behavioral factors, such as smoking and alcohol use are related to increased exposure to ROS. ${ }^{46,47}$ 8-OHdG has been reported to be positively associated with a range of sociodemographic and lifestyle determinants, such as age and cigarette exposure..$^{48}$ Dysregulations of the hypothalamic-pituitary-adrenal axis have been reported in depression, and these could be contributing factors to increased oxidative stress. ${ }^{7,27}$ It is already known that ischemic stroke activates the inflammatory pathway, and
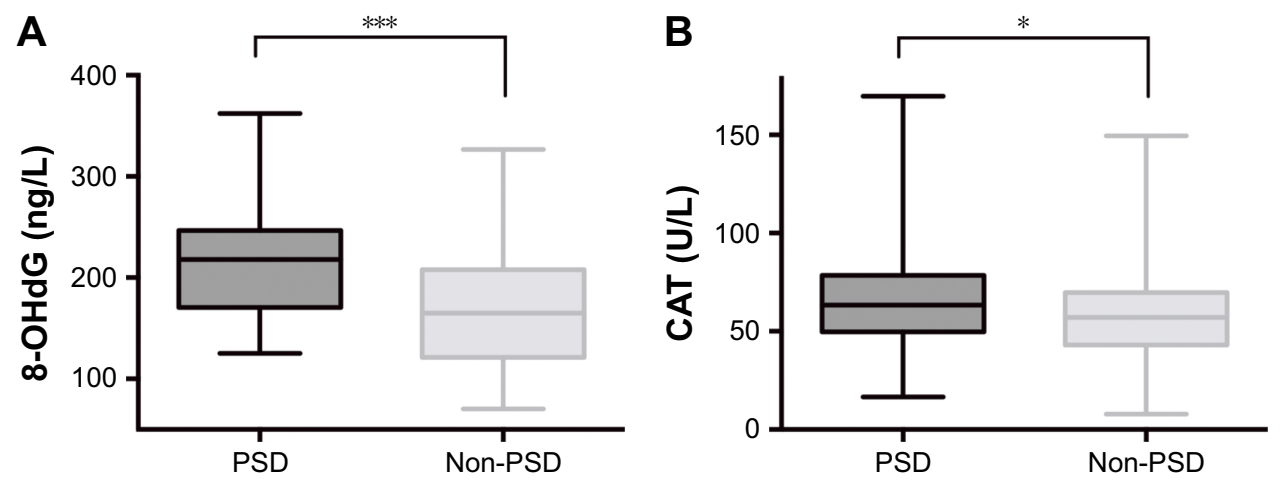

Figure I Serum levels of 8-OHdG and CAT in patients with PSD and those without depression.

Notes: (A) 8-OHdG levels, (B) CAT levels. In the box-and-whisker plots, the horizontal line in the middle of each box indicates the median value; the lower and upper ends of the box represent the 25 th and 75 th percentiles, respectively, and the peripheral lines extending to the outer fences represent 10 th and 90 th percentiles. $* P<0.05$; $* * * P<0.00$ I compared with the non-PSD group via Mann-Whitney $U$ test.

Abbreviations: 8-OHdG, 8-hydroxy-2'-deoxyguanosine; CAT, catalase; PSD, post-stroke depression. 

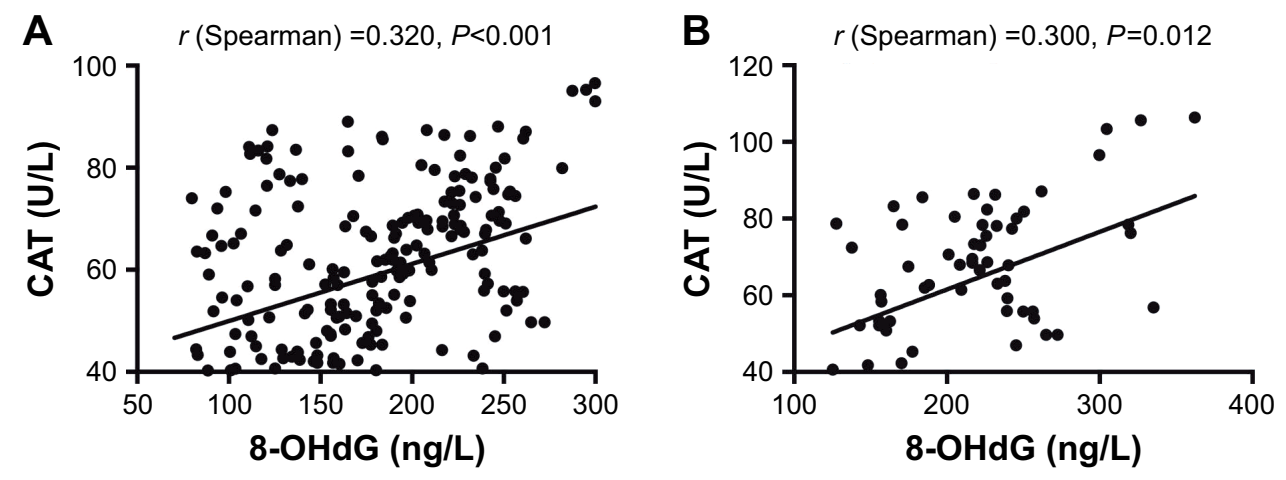

Figure 2 Correlation between CAT and 8-OHdG levels in acute stroke patients or PSD patients. Note: (A) In stroke patients, (B) in PSD patients.

Abbreviations: 8-OHdG, 8-hydroxy-2'-deoxyguanosine; CAT, catalase; PSD, post-stroke depression.

pro-inflammatory cytokines, in turn, amplify the oxidative stress response. ${ }^{49}$ Moreover, recent studies have revealed that exogenous $8-\mathrm{OHdG}$ could be a functional molecule in the regulation of oxidative stress-induced gastritis through binding or blocking the related signals responsible for the gastric inflammatory cascade. The brain, characterized by high oxygen consumption and low antioxidant defenses, is particularly vulnerable to oxidative damage. Oxidative DNA damage has been identified as an important contributor to neurodegeneration associated with acute central nervous system injuries and neurological diseases. In particular, unchecked oxidative DNA damage has been found to be associated with the activation of a series of signaling events, such as p53 and poly(ADP-ribose) polymerase-1, which have been shown to promote neuronal loss following

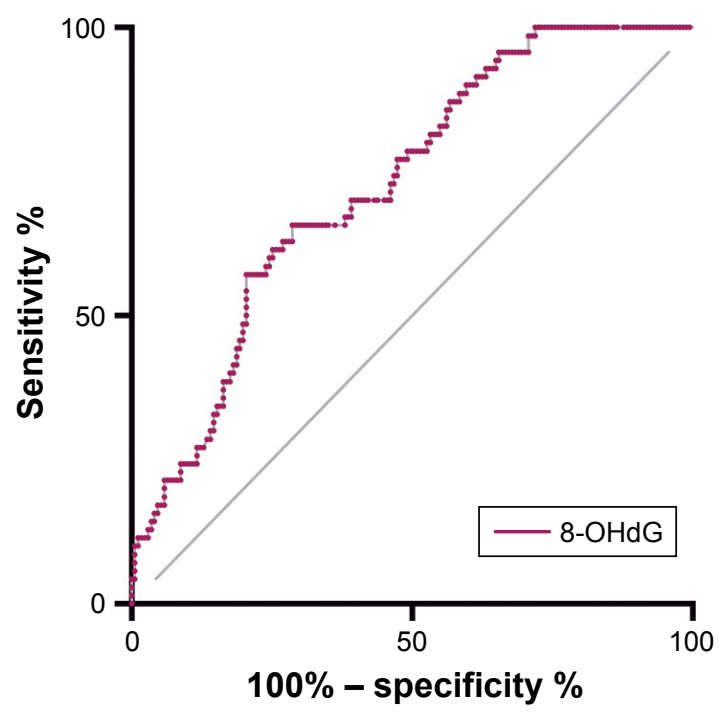

Figure 3 Receiver operator characteristic curve demonstrating sensitivity as a function of I-specificity for predicting post-stroke depression within I month based on the serum 8-OHdG levels in stroke patients.

Abbreviation: 8-OHdG, 8-hydroxy-2'-deoxyguanosine. ischemic stroke. ${ }^{50}$ There is some evidence from post-mortem studies that has suggested that $8-\mathrm{OHdG}$ levels increased in depressed patients. ${ }^{51}$

Another important finding of our study was that serum 8-OHdG levels were positively correlated with serum CAT levels. In our study, serum CAT levels increased in depressed patients. The increased CAT levels might be a compensatory mechanism for the excessive production of ROS in depressed patients during the acute phase. Galecki et al also found that major depressive disorder (MDD) patients had significantly higher red blood cell CAT activities than healthy controls during acute depressive episodes. ${ }^{52}$ Our results were in line with the recent evidence supporting that increased serum CAT levels might be indicators of acute depressive episodes in MDD patients. ${ }^{8}$

There were several limitations to our study. First, we excluded patients with severe comprehension deficits or a history of stroke, which may have added some bias to the results. Second, the serum 8-OHdG levels were evaluated only once at the time of admission. It is worth investigating whether there are longitudinal changes in serum 8-OHdG levels and the relationship between these results and PSD. Third, we did not compare the 8-OHdG levels in serum with those in the urine. Fourth, the study subjects came from only 1 clinic; thus, it would be inappropriate to generalize the findings.

\section{Conclusion}

In summary, in spite of these limitations, our study demonstrated an important association between serum 8-OHdG levels at admission and the development of PSD 1 month after stroke. Stroke patients should be monitored for high 8-OHdG levels and followed up for appropriate interventions. Additional studies are needed to confirm this association, which may provide new proposals for the development of PSD. 


\section{Acknowledgments}

This study was supported by the National Key Technology Research and Development Program of the Ministry of Science and Technology of China (grant number: 2015BAI13B01), National Key Technology R\&D Program in the 11th Fiveyear Plan of China (grant number: 2009BAI77B06), and Wenzhou Municipal Sci-Tech Bureau Program (grant number: Y20160002).

\section{Author contributions}

$\mathrm{JH}$ designed the study and wrote the protocol. ZL and YC conducted literature searches and provided summaries of previous research studies. ZL conducted the statistical analysis and wrote the first draft of the manuscript. JH took responsibility for the integrity of the data. All authors contributed toward data analysis, drafting and revising the paper and agree to be accountable for all aspects of the work. All authors reviewed and approved the final version of the manuscript.

\section{Disclosure}

The authors report no conflicts of interest in this work.

\section{References}

1. Ojagbemi A, Akinyemi R, Baiyewu O. Cognitive dysfunction and functional limitations are associated with major depression in stroke survivors attending rehabilitation in Nigeria. NeuroRehabilitation. 2014;34(3):455-461.

2. Williams LS, Ghose SS, Swindle RW. Depression and other mental health diagnoses increase mortality risk after ischemic stroke. $\mathrm{Am} \mathrm{J}$ Psychiatry. 2004;161(6):1090-1095.

3. Hackett ML, Pickles K. Part I: frequency of depression after stroke: an updated systematic review and meta-analysis of observational studies. Int J Stroke. 2014;9(8):1017-1025.

4. Canugovi C, Misiak M, Ferrarelli LK, Croteau DL, Bohr VA. The role of DNA repair in brain related disease pathology. DNA Repair (Amst). 2013;12(8):578-587.

5. Xu Y, Wang C, Klabnik JJ, O’Donnell JM. Novel therapeutic targets in depression and anxiety: antioxidants as a candidate treatment. Curr Neuropharmacol. 2014;12(2):108-119.

6. Cichon N, Bijak M, Miller E, Niwald M, Saluk J. Poststroke depression as a factor adversely affecting the level of oxidative damage to plasma proteins during a brain stroke. Oxid Med Cell Longev. 2015; 2015:408745.

7. Lopresti AL, Maker GL, Hood SD, Drummond PD. A review of peripheral biomarkers in major depression: the potential of inflammatory and oxidative stress biomarkers. Prog Neuropsychopharmacol Biol Psychiatry. 2014;48:102-111.

8. Tsai MC, Huang TL. Increased activities of both superoxide dismutase and catalase were indicators of acute depressive episodes in patients with major depressive disorder. Psychiatry Res. 2016;235:38-42.

9. Milanlioglu A, Aslan M, Ozkol H, Cilingir V, Nuri Aydin M, Karadas S. Serum antioxidant enzymes activities and oxidative stress levels in patients with acute ischemic stroke: influence on neurological status and outcome. Wien Klin Wochenschr. 2016;128(5-6):169-174.

10. Cojocaru IM, Cojocaru M, Sapira V, Ionescu A. Evaluation of oxidative stress in patients with acute ischemic stroke. Rom J Intern Med. 2013; 51(2):97-106.
11. Takemori K, Murakami T, Kometani T, Ito H. Possible involvement of oxidative stress as a causative factor in blood-brain barrier dysfunction in stroke-prone spontaneously hypertensive rats. Microvasc Res. 2013;90:169-172.

12. Radi E, Formichi P, Battisti C, Federico A. Apoptosis and oxidative stress in neurodegenerative diseases. J Alzheimers Dis. 2014;42(Suppl 3): S125-S152.

13. Hsieh YW, Lin KC, Korivi M, Lee TH, Wu CY, Wu KY. The reliability and predictive ability of a biomarker of oxidative DNA damage on functional outcomes after stroke rehabilitation. Int J Mol Sci. 2014; 15(4):6504-6516

14. Xiang F, Shuanglun X, Jingfeng W, et al. Association of serum 8-hydroxy-2'-deoxyguanosine levels with the presence and severity of coronary artery disease. Coron Artery Dis. 2011;22(4):223-227.

15. Hsieh YW, Lin KC, Korivi M, Lee TH, Wu CY, Wu KY. The reliability and predictive ability of a biomarker of oxidative DNA damage on functional outcomes after stroke rehabilitation. Int J Mol Sci. 2014; 15(4):6504-6516.

16. Liu H, Uno M, Kitazato KT, et al. Peripheral oxidative biomarkers constitute a valuable indicator of the severity of oxidative brain damage in acute cerebral infarction. Brain Res. 2004;1025(1-2):43-50.

17. Savic-Radojevic A,Pljesa-ErcegovacM,MaticM,SimicD, RadovanovicS, Simic T. Novel biomarkers of heart failure. Adv Clin Chem. 2017;79: 93-152.

18. Gmitterova K, Heinemann U, Gawinecka J, et al. 8-OHdG in cerebrospinal fluid as a marker of oxidative stress in various neurodegenerative diseases. Neurodegener Dis. 2009;6(5-6):263-269.

19. Simsek S, Gencoglan S, Yuksel T, Kaplan I, Alaca R, Aktas H. Oxidative stress and DNA damage in untreated first-episode psychosis in adolescents. Neuropsychobiology. 2016;73(2):92-97.

20. Brea D, Roquer J, Serena J, Segura T, Castillo J; ARTICO STUDY. Oxidative stress markers are associated to vascular recurrence in noncardioembolic stroke patients non-treated with statins. BMC Neurol. 2012;12(1):65

21. Chen YC, Chen CM, Liu JL, Chen ST, Cheng ML, Chiu DT. Oxidative markers in spontaneous intracerebral hemorrhage: leukocyte 8-hydroxy2 -deoxyguanosine as an independent predictor of the 30-day outcome. J Neurosurg. 2011;115(6):1184-1190.

22. Myoren T, Kobayashi S, Oda S, et al. An oxidative stress biomarker, urinary 8-hydroxy-2'-deoxyguanosine, predicts cardiovascular-related death after steroid therapy for patients with active cardiac sarcoidosis. Int J Cardiol. 2016;212:206-213.

23. Camkurt MA, Findikli E, Izci F, Kurutas EB, Tuman TC. Evaluation of malondialdehyde, superoxide dismutase and catalase activity and their diagnostic value in drug naive, first episode, non-smoker major depression patients and healthy controls. Psychiatry Res. 2016;238:81-85.

24. Szebeni A, Szebeni K, Diperi T, et al. Shortened telomere length in white matter oligodendrocytes in major depression: potential role of oxidative stress. Int J Neuropsychopharmacol. 2014;17(10):1579-1589.

25. Black CN, Bot M, Scheffer PG, Penninx BW. Oxidative stress in major depressive and anxiety disorders, and the association with antidepressant use; results from a large adult cohort. Psychol Med. 2017; 47(5):936-948.

26. Hirose A, Terauchi M, Akiyoshi M, Owa Y, Kato K, Kubota T. Depressive symptoms are associated with oxidative stress in middleaged women: a cross-sectional study. Biopsychosoc Med. 2016;10:12.

27. Black CN, Bot M, Scheffer PG, Cuijpers P, Penninx BW. Is depression associated with increased oxidative stress? A systematic review and meta-analysis. Psychoneuroendocrinology. 2015;51:164-175.

28. Fei K, Benn EK, Negron R, Arniella G, Tuhrim S, Horowitz CR. Prevalence of depression among stroke survivors: racial-ethnic differences. Stroke. 2016;47(2):512-515.

29. Muller MJ, Dragicevic A. Standardized rater training for the Hamilton Depression Rating Scale (HAMD-17) in psychiatric novices. $J$ Affect Disord. 2003;77(1):65-69.

30. Hamilton M. A rating scale for depression. J Neurol Neurosurg Psychiatry. 1960;23(1):56-62. 
31. Frank E, Prien RF, Jarrett RB, et al. Conceptualization and rationale for consensus definitions of terms in major depressive disorder. Arch Gen Psychiatry. 1991;48(9):851-855.

32. Naarding P, Leentjens AF, Van KF, Verhey FR. Disease-specific properties of the rating scale for depression in patients with stroke, Alzheimer's dementia, and Parkinson's disease. J Neuropsychiatry Clin Neurosci. 2002;14(3):329-334.

33. Mula M, Iudice A, La Neve A, et al. Validation of the Hamilton Rating Scale for depression in adults with epilepsy. Epilepsy Behav. 2014; 41:122-125

34. Schwarzbold ML, Diaz AP, Nunes JC, et al. Validity and screening properties of three depression rating scales in a prospective sample of patients with severe traumatic brain injury. Rev Bras Psiquiatr. 2014; 36(3):206-212.

35. Lobbestael J, Leurgans M, Arntz A. Inter-rater reliability of the structured clinical interview for DSM-IV Axis I Disorders (SCID I) and Axis II Disorders (SCID II). Clin Psychol Psychother. 2011;18(1):75-79.

36. Fischer U, Baumgartner A, Arnold M, et al. What is a minor stroke? Stroke. 2010;41(4):661-666.

37. Luengo-Fernandez R, Gray AM, Rothwell PM. Effect of urgent treatment for transient ischaemic attack and minor stroke on disability and hospital costs (EXPRESS study): a prospective population-based sequential comparison. Lancet Neurol. 2009;8(3):235-243.

38. Brott T, Adams HP Jr, Olinger CP, et al. Measurements of acute cerebral infarction: a clinical examination scale. Stroke. 1989;20(7):864-870.

39. Mahoney FI, Barthel DW. Functional evaluation: the Barthel Index. Md State Med J. 1965;14:61-65.

40. Folstein MF, Robins LN, Helzer JE. The mini-mental state examination. Arch Gen Psychiatry. 1983;40(7):812.

41. Ayerbe L, Ayis S, Crichton S, Wolfe CD, Rudd AG. The natural history of depression up to 15 years after stroke: the South London Stroke Register. Stroke. 2013;44(4):1105-1110.

42. Matsuzaki S, Hashimoto M, Yuki S, Koyama A, Hirata Y, Ikeda M. The relationship between post-stroke depression and physical recovery. J Affect Disord. 2015;176:56-60.
43. Forlenza MJ, Miller GE. Increased serum levels of 8-hydroxy$2^{\prime}$-deoxyguanosine in clinical depression. Psychosom Med. 2006; 68(1):1-7.

44. Vargas HO, Nunes SO, Pizzo de Castro M, et al. Oxidative stress and lowered total antioxidant status are associated with a history of suicide attempts. J Affect Disord. 2013;150(3):923-930.

45. Iida T, Inoue K, Ito $\mathrm{Y}$, et al. Comparison of urinary levels of 8-hydroxy2 '-deoxyguanosine between young females with and without depressive symptoms during different menstrual phases. Acta Med Okayama. 2015;69(1):45-50.

46. Li H, Horke S, Forstermann U. Oxidative stress in vascular disease and its pharmacological prevention. Trends Pharmacol Sci. 2013;34(6): 313-319.

47. Vargas HO, Nunes SO, de Castro MR, et al. Oxidative stress and inflammatory markers are associated with depression and nicotine dependence. Neurosci Lett. 2013;544:136-140.

48. Black CN, Bot M, Scheffer PG, Penninx BW. Sociodemographic and lifestyle determinants of plasma oxidative stress Markers 8-OHdG and F2-Isoprostanes and associations with metabolic syndrome. Oxid Med Cell Longev. 2016;2016:7530820.

49. Xu G, Luo K, Liu H, Huang T, Fang X, Tu W. The progress of inflammation and oxidative stress in patients with chronic kidney disease. Ren Fail. 2015;37(1):45-49.

50. Smith JA, Park S, Krause JS, Banik NL. Oxidative stress, DNA damage, and the telomeric complex as therapeutic targets in acute neurodegeneration. Neurochem Int. 2013;62(5):764-775.

51. Michel TM, Pulschen D, Thome J. The role of oxidative stress in depressive disorders. Curr Pharm Des. 2012;18(36):5890-5899.

52. Galecki P, Szemraj J, Bienkiewicz M, Florkowski A, Galecka E. Lipid peroxidation and antioxidant protection in patients during acute depressive episodes and in remission after fluoxetine treatment. Pharmacol Rep. 2009;61(3):436-447. 


\section{Supplementary materials}

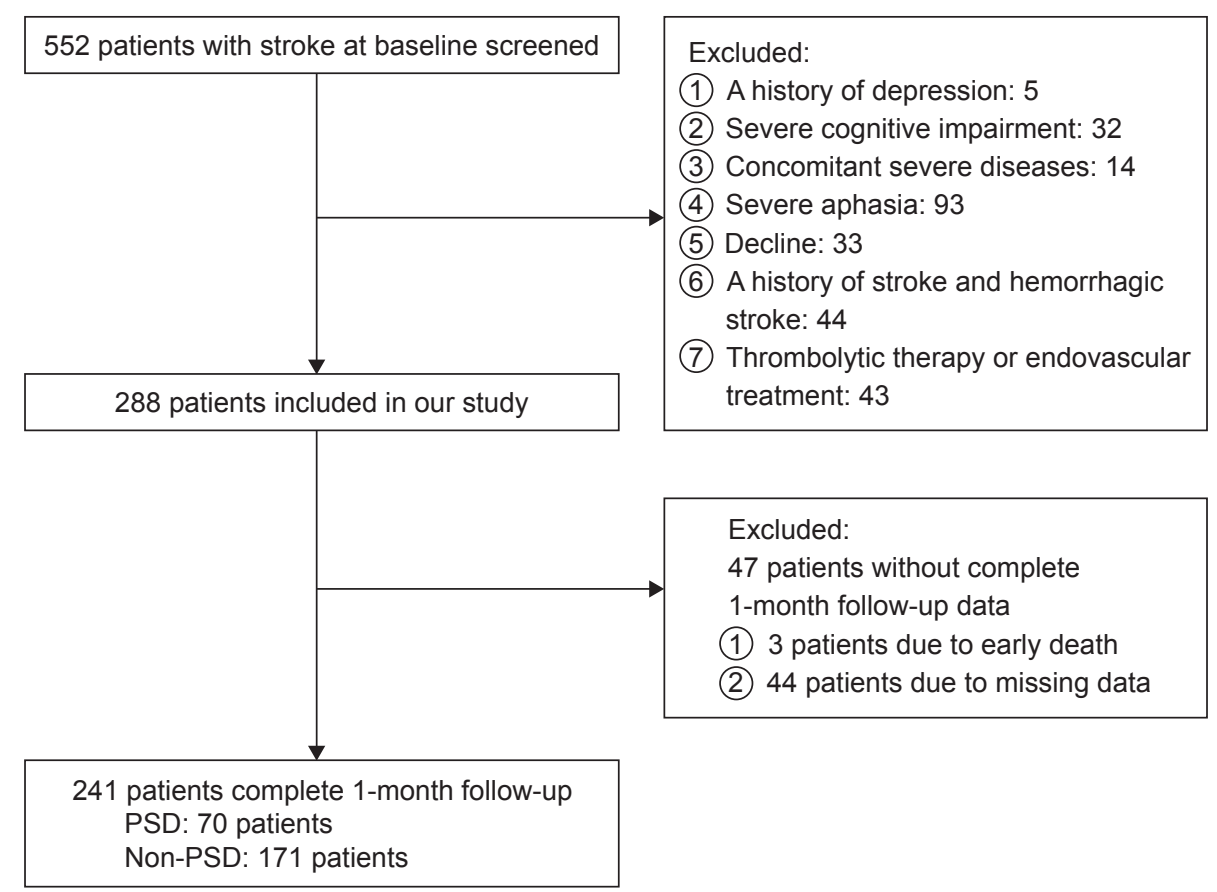

Figure SI Study flow chart.

Abbreviation: PSD, post-stroke depression.

Table SI Prescribed drugs employed of the studied subjects at admission

\begin{tabular}{|c|c|c|c|}
\hline Drugs & $\begin{array}{l}\text { PSD patients } \\
(\mathrm{n}=70)\end{array}$ & $\begin{array}{l}\text { Non-PSD patients } \\
(n=|7|)\end{array}$ & $P$-value \\
\hline Statins use, $\mathrm{n}(\%)$ & $69(98.6)$ & $164(95.9)$ & 0.514 \\
\hline $\begin{array}{l}\text { Alprostadil injection, cinepazide maleate injection, or } \\
\text { vinpocetine injection }\end{array}$ & $12(17.1)$ & $42(24.6)$ & 0.210 \\
\hline Aspirin or clopidogrel use, $n$ (\%) & $65(92.9)$ & $158(92.4)$ & 0.902 \\
\hline Hypertension medicine use, $\mathrm{n}(\%)$ & $36(5 \mathrm{I} .4)$ & $79(46.2)$ & 0.461 \\
\hline Diabetes medicine use, $\mathrm{n}(\%)$ & $19(27.1)$ & $45(26.3)$ & 0.895 \\
\hline Butylphthalide use, n (\%) & $24(34.3)$ & $68(39.8)$ & 0.427 \\
\hline Vitamin C injection use, $\mathrm{n}(\%)$ & $4(5.7)$ & $12(7.0)$ & 0.933 \\
\hline Mannitol use, n (\%) & $3(4.3)$ & $5(2.9)$ & 0.889 \\
\hline Ginkgo leaf extract and dipyridamole injection use, n (\%) & $4(5.7)$ & $10(5.8)$ & 1.000 \\
\hline Urinary kallidinogenase use, $\mathrm{n}(\%)$ & $\mathrm{I}(\mathrm{I} .4)$ & $8(4.7)$ & 0.424 \\
\hline Folic acid use, n (\%) & $3(4.3)$ & $7(4.1)$ & 1.000 \\
\hline
\end{tabular}

Abbreviation: PSD, post-stroke depression.

Table S2 Serum 8-OHdG and CAT levels according to the severity of stroke and depression

\begin{tabular}{|c|c|c|c|c|c|}
\hline Laboratory variables & $A(n=130)$ & $B(n=34)$ & $C(n=4 I)$ & $D(n=36)$ & $P$-value \\
\hline 8-OHdG (ng/L), median (IQR) & $163.9(|24.8-2| 1.1)$ & $223.1 * * *(185.4-258.8)$ & $164.9 \ldots$ & $212.9 * * *, a$ & $<0.001 * * *$ \\
\hline CAT (U/L), median (IQR) & $57.1(42.9-70.7)$ & $64.6(45.1-77.6)$ & $56.8(43.1-69.1)$ & $63.4(5 \mathrm{I} .2-80.4)$ & 0.150 \\
\hline
\end{tabular}

Notes: Patients were divided into 4 groups according to the severity of stroke and depression. A group: minor stroke (NIHSS $\leq 3)+$ depression $(-)$; $B$ group: minor stroke $($ NIHSS $\leq 3)+$ depression (+); C group: moderate-to-severe stroke (NIHSS $>4)+$ depression $(-)$; D group: moderate-to-severe stroke (NIHSS $>4)+$ depression $(+)$. The $P$-values reflect comparisons of serum $8-O H d G$ and CAT levels in the 4 groups. ${ }^{*} * * P<0.00$ I compared to A group by univariate analysis; ${ }^{\#} P<0.00 \mathrm{I}$ compared to $B$ group by univariate analysis; ${ }^{a} P<0.001$ compared to $C$ group by univariate analysis.

Abbreviations: 8-OHdG, 8-Hydroxy-2'-deoxyguanosine; CAT, catalase; IQR, interquartile range; NIHSS, National Institutes of Health Stroke Scale. 
Table S3 Comparisons of serum 8-OHdG and CAT levels in patients with minor stroke and those with moderate-to-severe stroke

\begin{tabular}{llll}
\hline Laboratory variables & $\begin{array}{l}\text { NIHSS } \leq \mathbf{3} \\
(\mathbf{n}=\mathbf{1 6 4})\end{array}$ & $\begin{array}{l}\text { NIHSS }>\mathbf{3} \\
(\mathbf{n}=\mathbf{7 7})\end{array}$ & $\boldsymbol{P}$-value \\
\hline 8-OHdG (ng/L), mean \pm SD & $182.5 \pm 60.6$ & $184.1 \pm 58.4$ & 0.841 \\
CAT (U/L), mean \pm SD & $58.8 \pm 21.9$ & $60.2 \pm 20.4$ & 0.638 \\
\hline
\end{tabular}

Abbreviations: 8-OHdG, 8-Hydroxy-2'-deoxyguanosine; CAT, catalase; NIHSS, National Institutes of Health Stroke Scale.

Table S4 Correlations between oxidative stress markers and NIHSS scores

\begin{tabular}{llr}
\hline NIHSS scores & 8-OHdG & CAT \\
\hline NIHSSI & $r=-0.03, P=0.642$ & $r=0.04 I, P=0.523$ \\
NHSS2 & $r=-0.219, P=0.068$ & $r=0.090, P=0.458$ \\
NIHSS3 & $r=-0.086, P=0.265$ & $r=-0.030, P=0.700$ \\
\hline
\end{tabular}

Notes: NIHSSI: stroke group. NIHSS2: post-stroke depression group. NIHSS3: non-PSD group.

Abbreviations: 8-OHdG, 8-Hydroxy-2'-deoxyguanosine; CAT, catalase; NIHSS, National Institutes of Health Stroke Scale.

\section{Publish your work in this journal}

Neuropsychiatric Disease and Treatment is an international, peerreviewed journal of clinical therapeutics and pharmacology focusing on concise rapid reporting of clinical or pre-clinical studies on a range of neuropsychiatric and neurological disorders. This journal is indexed on PubMed Central, the 'PsycINFO' database and CAS, and is the official journal of The International Neuropsychiatric Association (INA). The manuscript management system is completely online and includes a very quick and fair peer-review system, which is all easy to use. Visit http://www.dovepress.com/testimonials.php to read real quotes from published authors.

\footnotetext{
Submit your manuscript here: http://www.dovepress.com/neuropsychiatric-disease-and-treatment-journal
} 